\title{
Initial Experience and Analysis of Outcomes Using the Visualized Percutaneous Endoscopic Lumbar Discectomy Technique for the Treatment of Lumbar Disc Herniation
}

\author{
Lei KONG ${ }^{*}$, Li-Qun DUAN ${ }^{2}$, Hong-Guang $X^{3}$ \\ ${ }^{1}$ Medical College of Shandong University, Jinan 250000, Shandong Province, People's Republic of China \\ ${ }^{2}$ The First Affiliated Hospital of University of Science and Technology of China, Department of Spine Orthopedic, Hefei 230001, Anhui Province, \\ People's Republic of China \\ ${ }^{3}$ The First Affiliated Hospital of WanNan Medical College, Department of Spine Orthopedic, WuHu 241000, Anhui Province, People's Republic \\ of China \\ *The author contributed equally to this work and should be considered co-first author.
}

Corresponding author: Hong-Guang XU 80723269@qq.com

\section{ABSTRACT}

AIM: To introduce a visual surgical concept using a retrospective comparison of the outcomes between visualized percutaneous endoscopic lumbar discectomy (V-PELD) and conventional PELD techniques.

MATERIAL and METHODS: Data from 61 patients in the conventional PELD group and 55 in the V-PELD group. Data included the duration of the operation, number of intraoperative radiation exposure events, and follow-up information for 24 months. The Oswestry Disability Index (ODI) and visual analog scale (VAS) scores were collected.

RESULTS: The mean ( \pm SD) operating time for the V-PELD group was $57.82 \pm 11.25$ min, and the mean duration of radiation exposure was $0.74 \pm 0.32 \mathrm{~min}$. The mean operating time for the conventional group was $63.16 \pm 14.49 \mathrm{~min}(p<0.05)$, and the mean duration of radiation exposure was $2.81 \pm 1.12 \mathrm{~min}(\mathrm{p}<0.001)$. All patients in both groups demonstrated significant improvement in the ODI and VAS scores after surgery $(\mathrm{p}<0.05)$.

CONCLUSION: V-PELD is a minimally invasive surgical technique that involves less radiation exposure and is more efficient for treating lumbar disc herniation.

KEYWORDS: Visualized, Percutaneous endoscopic lumbar discectomy, Lumbar disc herniation

ABBREVIATIONS: V-PELD: Visualized percutaneous endoscopic lumbar discectomy, PELD: Percutaneous endoscopic lumbar discectomy, VAS: Visual analog scale, ODI: Oswestry Disability Index, SAP: Superior articular process

\section{INTRODUCTION}

I n China, modern-day, busy lifestyles require that patients recover quickly from disease treatment. As a result, increasing attention is being devoted to the concept of minimally invasive spinal surgery. Percutaneous endoscopic lumbar discectomy (PELD) is a representative surgical technique for treating lumbar disc herniation, and is well established and has been standardized $(1,2)$. Conventional open nucleus pulposus excision, however, involves severe tissue damage; as such, the use of microsurgical techniques has significantly reduced postsurgical sequalae (12). In recent years, technological advances in microsurgical techniques have been very rapid, and are inseparable from the progress of surgical technology in the development of optics and 
improvement in surgical instruments (21). With continuous advances in technology and methods, lumbar and cervical disc herniation or nerve root canal stenosis can now be treated using the minimally invasive PELD technique $(12,18,19)$. However, the high number of intraoperative punctures while under local anesthesia, which increases the risk for infection and abdominal visceral injury or hematoma (5), the increased exposure of patients and surgeons to radiation (16), especially beginners, and longer operation times have become difficult problems. The aim of the present article is to introduce and compare the V-PELD technique with the conventional PELD method in the treatment of lumbar disc herniation, specifically for superior articular process (SAP) plasty using directly visualized endoscopic assistance. Direct visualization decreases the number of punctures and shortens the operation time, which is important in reducing the risk for nerve root injury.

\section{MATERIAL and METHODS}

A total of 116 patients, who were treated between January 2016 and June 2017, were retrospectively analyzed. Sixty-one of these patients (12 female, 49 male; mean age, 39.1 years [range, 19-78 years]) underwent conventional PELD surgery. Forty-three patients underwent surgery at the L5/S1 level, and 18 at the L4/5 level. Fifty-five patients ( 11 female, 44 male, mean age, 38.6 years [range, 18-67 years]) were included in the V-PELD group, of whom 39 underwent surgery at the L5/S1 level and 16 at the L4/5 level (Table I). Patients were treated using the PELD technique by a team led by a single surgeon. The operation time and frequency of intraoperative radiographic exposure were recorded. Postoperative leg and back visual analog scale (VAS) scores and Oswestry Disability Index (ODI) were recorded and followed up at 3, 6, 12, and 24 months after surgery.

Table I: Demographic Parameters of the Patients

\section{Inclusion and Exclusion Criteria}

Patients included in the present study fulfilled the following criteria: X-ray imaging without lumbar instability; magnetic resonance or computed tomography imaging indicating lumbar disc herniation; not receiving other minimally invasive treatments; presence of more symptoms of leg pain than back pain, inefficacy of conservative therapy (lying on bed traction, physiotherapy) for at least six weeks; and necessity for foraminoplasty during surgery. Chronic low back pain, relevant levels of intervertebral foramen stenosis, degenerative lumbar instability with spondylolisthesis > grade I, or scoliosis $>20^{\circ}$, spinal instability and scoliosis causing loss of foraminal height. Patients in whom open surgery was required due to failed previous PELD surgery or those with cauda equina syndrome caused by protruding discs were excluded. In short, contraindication to PELD was the need for open fusion surgery $(11,12)$.

\section{Surgical Techniques}

Patients were positioned prone on a lumbar bridge before surgery. A marker was used to indicate the midline and the height of the iliac crest, which were delineated from preoperative assessment of axial magnetic resonance images to calculate the skin entry point, with appropriate adjustments according to patient body shape and weight. The L4/5 skin entry point was generally $10 \mathrm{~cm}$ from the midline and approximately $12 \mathrm{~cm}$ from the midline for the L5/S1 skin entry point (Figure 1A). Anesthesia was induced stepwise, and angular trajectories in the coronal plane (dorsal view) at the $L 4 / 5$ segment $\left(30-40^{\circ}\right)$ and the L5/S1 segment $\left(40-50^{\circ}\right)$ were used to plan transforaminal access. G-arm fluoroscopic guidance was performed to ensure the 18-gauge puncture needle reached the SAP of the vertebra. The final target point was the center of the caudal vertebral endplate on anteroposterior fluoroscopic view and the posterior edge line of the vertebral body in lateral fluoroscopic view (Figure

\begin{tabular}{|c|c|c|}
\hline Baseline characteristic & V-PELD & PELD \\
\hline Gender & $M(44) / F(11)$ & $M(49) / F(12)$ \\
\hline Age (years; median IQR) & $38.6(20.5)$ & $39.1(16.0)$ \\
\hline Postoperative severe complication, n (\%) & 0 & 0 \\
\hline Back pain & $2.0(3.6 \%)$ & $2(3.3 \%)$ \\
\hline Operation time (minuter) & $57.82 \pm 11.25$ & $63.16 \pm 14.49$ \\
\hline Radiation exposure time (min) & $0.74 \pm 0.32$ & $2.81 \pm 1.12$ \\
\hline Revision & 0 & $1(1.9 \%)$ \\
\hline
\end{tabular}

V-PELD: Visualized percutaneous endoscopic lumbar discectomy, PELD: Percutaneous endoscopic lumbar discectomy, M: Male, F: Female, L: Lumbar, IQR: Interquartile range. 

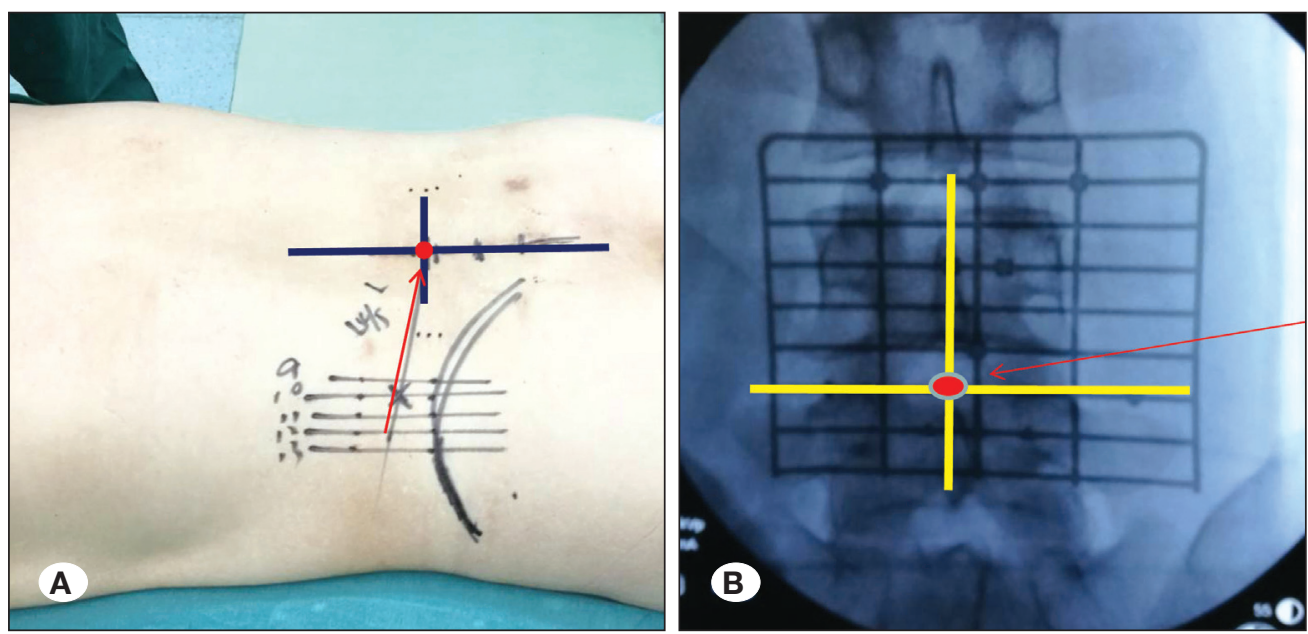

Figure 1: A) The surface midline as well as the extent of iliac crest marked using a pen. B) Planning the target puncture route.

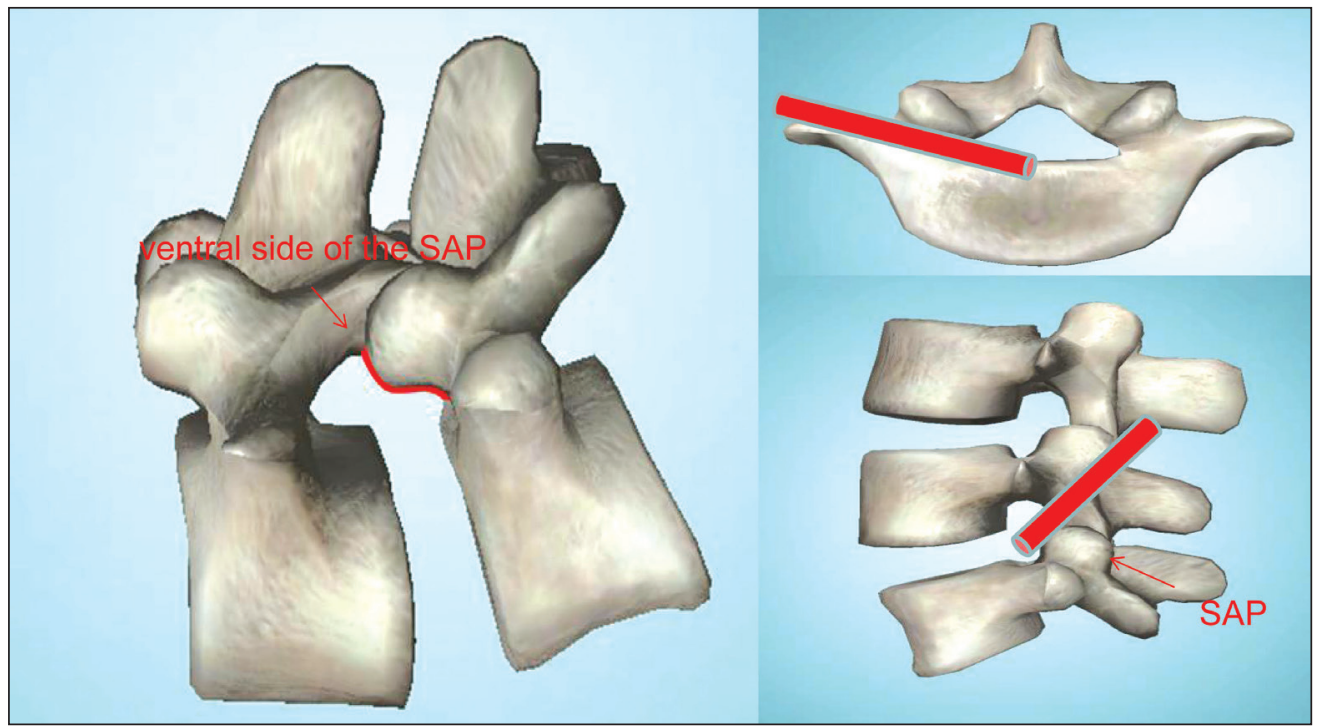

Figure 2: The ventral side of the superior articular process (SAP) and standard puncture route. Appropriate placement in the vertebral SAP was achieved.

$1 \mathrm{~B}, 2)$. Once it was confirmed that the needle reached the lateral portion of the SAP, more lidocaine was injected near the articular process to help relieve the patient's pain (4). The next step was to withdraw the needle a short distance and increase the ventral angle to advance the needle from the ventral side of the SAP into the foramen. The puncture needle was then removed and the guidewire was inserted to the ventral side of the SAP. An incision approximately $7 \mathrm{~mm}$ long was made and dilators were used to expand the soft tissue gradually along the guidewire. The working cannulas were then placed on the surface and lateral side of the SAP (Figure 3B). The SAP was identified directly along the dorsal side of the guidewire under endoscopic assistance. Working cannulas $(8.4 \mathrm{~mm})$, designed by the authors (Patent number: ZL 201720025370. $X$ ) were inserted, as was a $7.5 \mathrm{~mm}$ trephine system (Figure $4 \mathrm{~A}, \mathrm{~B})$ so that a $6.3 \mathrm{~mm}$ lighting system could be used to perform the operation with endoscopic assistance. When soft tissue covering the SAP was first viewed under endoscope, a flexible bipolar radio frequency probe was used to clear the soft tissue to expose the SAP. The SAP was accurately located under endoscopic visualization. The guidewire was taken out to identify the tip of the SAP and foraminoplasty was performed along the ventral side of the SAP (Figure 4C). During foraminoplasty, the trephine was moved close to the ventral side of SAP in a clockwise manner. The SAP bone mass was removed (Figure 4D) and endoscopic observation was performed at this time. It was different from the need for repeated fluoroscopy and observation of patient response during the operation to confirm the position of the trephine or reamer, a partial SAP resection via endoscopic procedure was performed under direct endoscopic visualization. If nerve root exposure was not satisfactory, articular process plasty could be continued according to the surgical plan. The next step was to insert the dilators again, in which a standard $7.5 \mathrm{~mm}$ working cannula replaced the $8.4 \mathrm{~mm}$ cannula. The subsequent procedure for disc removal was no different from that performed in the conventional group.

In the conventional group, the puncture needle often required a guide needle to access the disc space, this usually required 
multiple fluoroscopies-especially for beginners-to adjust the puncture needle. The dilators then expanded the soft tissue gradually along the guidewire, and the TOM Shidi needle or trephine was positioned on the ventral side of the SAP. After intraoperative multiple fluoroscopy confirmed that positioning was satisfactory, the guidewire was inserted, and the reamer or trephine was gradually expanded to the bone channel along the guidewire. The next step required repeated fluoroscopy and observation of the patient's response during the maneuver to confirm the position of the trephine or reamer until the foraminoplasty was satisfactory (Figure 3A) because they often experienced pain during surgery when the reamer or trephine irritated the nerve root. The herniated disc was removed and decompression was discontinued when the patient's symptoms disappeared and the nerve root (viewed under the endoscope) pulsed strongly with respiration.

\section{Follow-up}

Data recording and follow-up were initiated on the next day, and 3, 6, 12, and 24 months thereafter in the outpatient clinic. ODI and VAS scores were calculated for back and leg pain.

\section{Statistical Analysis}

Data analysis was performed using SPSS version 16.0 (IBM Corporation, Chicago, IL, USA). Differences were evaluated using one-way analysis of variance with post hoc Bonferroni correction. Differences with $\mathrm{p}<0.05$ were considered to be statistically significant.

\section{RESULTS}

A 56-year-old male in the conventional group exhibited lumbar disc herniation combined with endplate inflammation preoperatively and underwent revision surgery four months after the initial surgery in another hospital due to persistent back pain. There were 115 patients enrolled after two years of follow-up. The mean $( \pm S D$ ) operating time was $57.82 \pm 11.25$ min (Figure 5A) in the V-PELD group, and the mean number
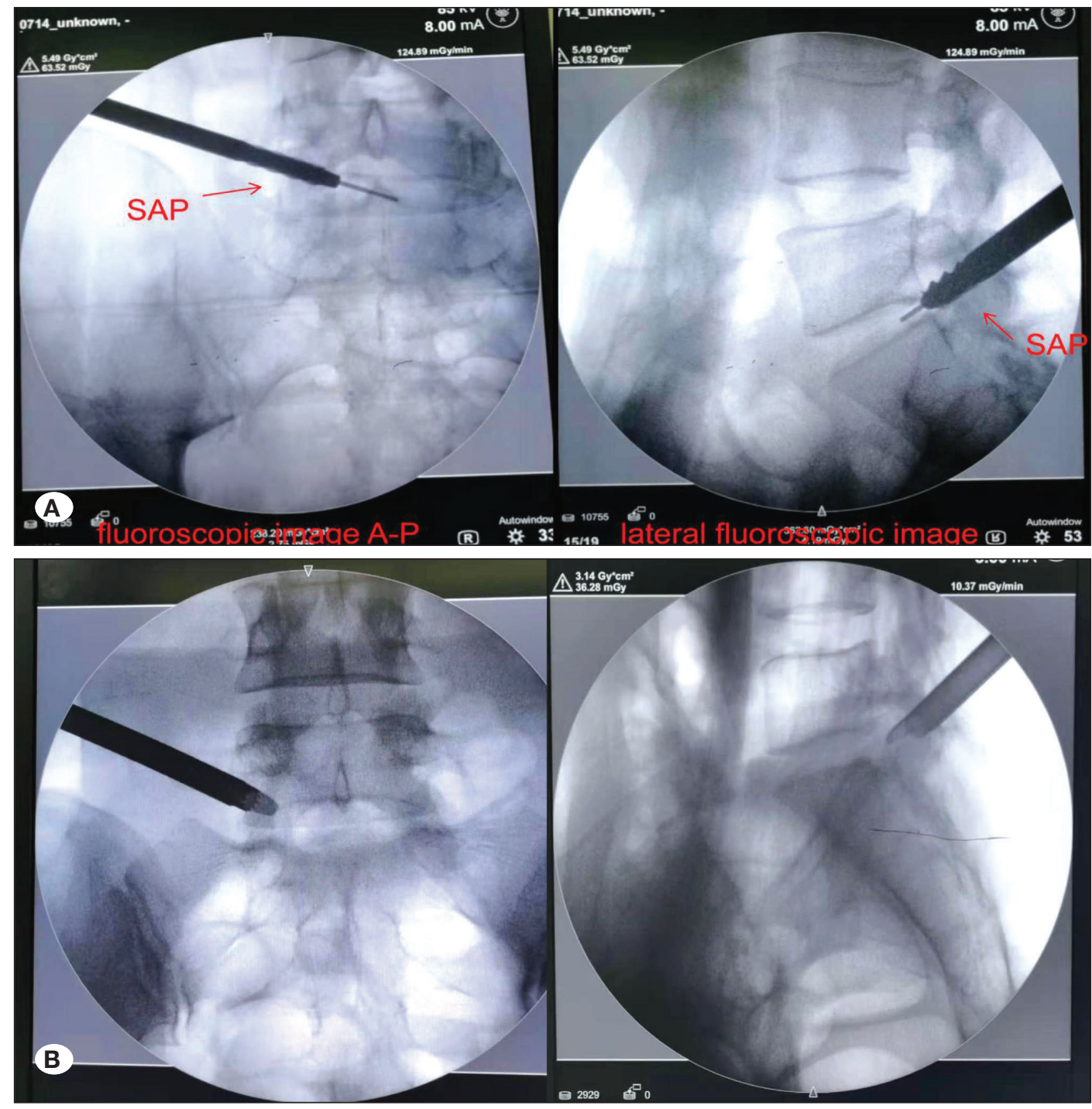

Figure 3: Conventional percutaneous endoscopic lumbar discectomy (PELD) (A), and visualized-PELD foraminoplasty under fluoroscopy (B). 

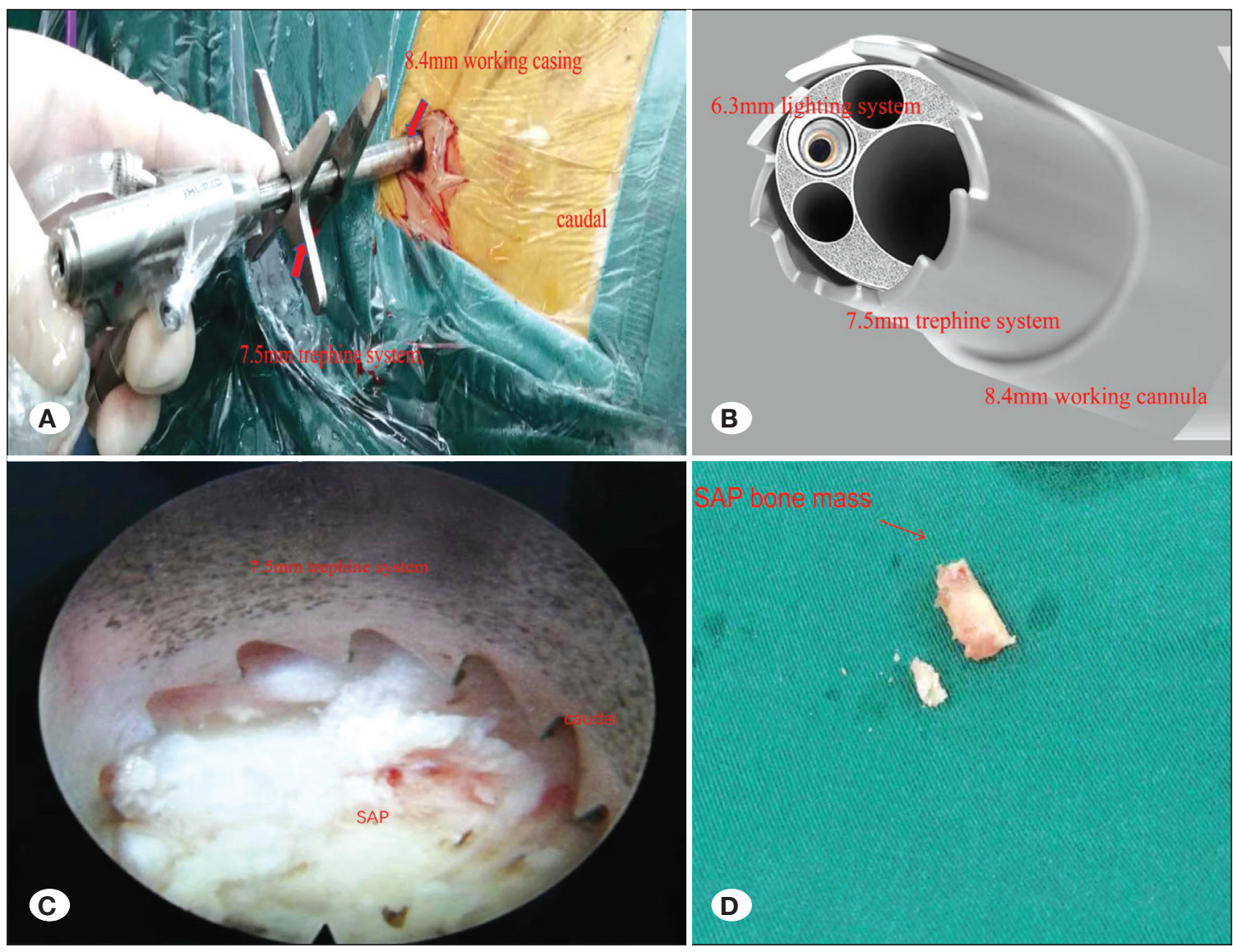

Figure 4:

A, B) Patented tools the authors designed are shown. The 8.4 $\mathrm{mm}$ working cannulas and the $7.5 \mathrm{~mm}$ trephine system.

C) An

intraoperative image of the operation.

D) The superior articular process bone mass was removed.

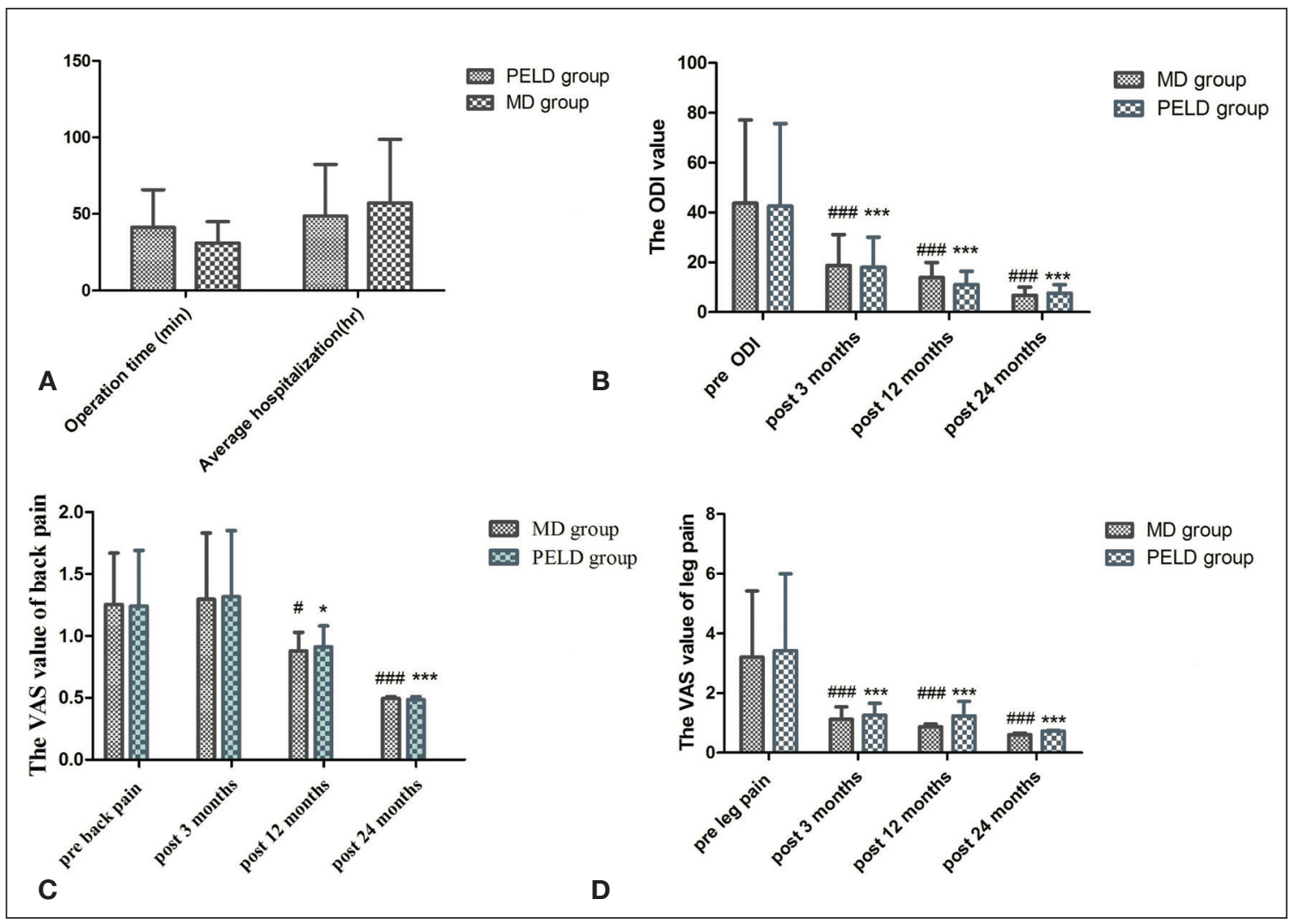

Figure 5:

A) Operating and radiation exposure times for the visualized percutaneous endoscopic lumbar discectomy (V-PELD) and conventional PELD groups. B) Pre- and postoperative Oswestry Disability Index (ODI) scores. C, D) Visual analog scale scores for preand postoperative leg and back pain. 
of radiation exposure events was $0.74 \pm 0.32$. The mean operating time in the conventional group was $63.16 \pm 14.49$ $\min (p<0.05) \mathrm{min}$, and the mean number of radiation exposure event was $2.81 \pm 1.12(p<0.001)$. Because continuous irrigation was performed during surgery and no drainage remained postoperatively, blood loss could not be precisely measured. Only one patient in the V-PELD group experienced postoperative transient dysesthesia and underwent rehabilitation and nerve stimulation treatment; the symptoms disappeared in $<2$ weeks. Three patients in the conventional group experienced transient leg dysesthesia postoperatively and underwent nerve stimulation treatment; their symptoms disappeared in $<6$ weeks. VAS back and leg pain scores are shown in Figure 5C and 5D, and pre- and postoperative ODI are shown in Figure 5B. The results demonstrated significant improvement in leg pain and ODI scores $(p<0.05)$. Two patients in the V-PELD group experienced low back pain 5 and 13 months after surgery, respectively. One of two patients in the conventional group experienced low back pain 6 months after surgery, while the other's low back pain increased on rainy days up to 24 months later. Overall, postoperative scores were significantly better than preoperative scores. All patients were treated after being admitted to the hospital, and the longest hospital stay for these patients was five days all were discharged the day after surgery.

\section{DISCUSSION}

For patients with lumbar disc herniation who fail conservative treatment, surgical removal of the nucleus pulposus and nerve root canal decompression are indispensable. Advances in technology have made possible full-endoscopic surgery with direct visualization and continuous irrigation for treating disc herniation. In elderly and infirmed individuals, local lidocaine and intravenous sedatives are used for anesthesia; as such, patients stay awake and avoid the risks associated with tracheal anesthesia (6,8-10).

The puncture target during the conventional PELD technique is the intervertebral disc (7). The most important and timeconsuming aspect of conventional PELD is the surgical puncture, which places the spinal needle exactly at the final target point while following the optimal trajectory (13). Soft tissue resistance causes the needle to deflect; as such, the needle usually cannot travel in the direction intended by the surgeon. The guide needle must be withdrawn first when redirection is required, then the surgeon needs to perform the puncture again. If not optimal, the needling procedure must be repeated (7). Endoscopic cannula placement in the inappropriate location is usually due to failure to place the spinal needle in the optimal target position, which always leads to inability to remove the disc herniation (13). In contrast, our V-PELD technique only requires us to reach the SAP. The entire SAP was the target of our intraoperative puncture, which means that our puncture changed from one point to one plane. We confirmed the puncture needle's arrival at the SAP using X-ray during the surgery, which means that we needed the total number of punctures during the operation. Compared with an average of 29 punctures with conventional fluoroscopy and standard instrumentation (17), the V-PELD technique significantly reduces the number of punctures needed by using fluoroscopic guidance. More importantly, our puncture target is the SAP and, as long as our puncture needle is on the SAP-regardless of whether it is the tip, the body or the root as visualized using an endoscope-we can adjust the trephine appropriately according to the needs of the surgery. The prerequisite behind this technique is that one must be familiar with the anatomy of the SAP under endoscopic visualization and to accurately locate the SAP using the endoscope, which is the key to the technology. For beginners, the puncture needle reached the ventral side of the SAP, and the tip of the SAP can be found directly along the dorsal side of the guidewire using the endoscope. For common disc herniation, removing a little bone from the SAP tip and ventral side is sufficient to adequately expose the nerve roots, which avoids removing excess bone under fluoroscopy. When the surgeon completes the foraminoplasty, an $8.4 \mathrm{~mm}$ cannula can be used to continue the operation; however, a transitional cannula may be added in the interim to prevent the sleeve and endoscopic working sleeve from loosening. The other option is to use a standard $7.5 \mathrm{~mm}$ working cannula that could be replaced.

After 24 months, the follow-up results of our endoscopic surgery patients were consistent with data reported by other investigators (19). The truth is that endoscopic surgery is not as "perfect" as initally imagined. Ahn reported a symptomatic dural tear rate of $1.1 \%$ in 816 consecutive cases. According to the authors, temporary dysesthesia is the most common complication, and the incidence of this complication is reported to be $2-6.53 \%$ (3). In the conventional group, the incidence of temporary dysesthesia was $4.9 \%$ and $1.8 \%$ in the V-PELD group. These postoperative symptoms are due to intraoperative stimulation of the dorsal root ganglion (3). Presently, there are issues with puncture types and navigation (20). While ultrasound-guided techniques are used to reduce radiation exposure and surgical risk, they also increase operative time and patient costs. It is undoubtedly advantageous to use a trephine for osteophytes and SAP foraminoplasty (14). Li invented a specially designed instrument for SAP foraminoplasty, and placed a graded duck-mouth protection cannula on the ventral side of the SAP to protect the nerve roots and dorsal root ganglion from the operating area stimulated by the trephine (15). However, it is necessary to confirm the position of the cannula and trephine during surgery using fluoroscopy. The advantages of the V-PELD technique and surgical operation under direct vision are apparent - they not only significantly reduce the number of fluoroscopy procedures but also avoid injuring the dural sac and irritating the nerve root during surgery.

We can perform foraminoplasty with excellent visualization and make timely adjustments during surgery. Even spinal canal stenosis and lateral recess stenosis procedures can be performed when nerve root decompression is needed. We advocate an efficient operation; as such, we designed an enlarged working sleeve to enable us to use the enlarged trephine system under visualization. Of course, foraminoplasty can also be performed using an endoscopic 
power system (such as a grinding drill) when the position of the SAP is confirmed using fluoroscopy during surgery. The $V$-PELD technique requires the surgeon to be familiar with the SAP and anatomical identification under endoscopic visualization. Although the learning curve is shorter compared with conventional technology, beginners should practice on cadavers and receive teaching demonstrations from senior surgeons.

\section{CONCLUSION}

Compared with the conventional technique, advantages of the V-PELD technique using specially designed instruments include less radiation exposure and fewer punctures during surgery. It was more effective and safer than conventional technology for the treatment of lumbar disc herniation during our 2 years of follow-up. However, with our small sample size and short follow-up time, we also need longer-term studies with larger sample sizes to demonstrate the benefits of the V-PELD technique in preventing complications.

\section{REFERENCES}

1. Ahn Y: Percutaneous endoscopic decompression for lumbar spinal stenosis. Expert Rev Med Devices 11:605-616, 2014

2. Ahn Y: Transforaminal percutaneous endoscopic lumbar discectomy: Technical tips to prevent complications. Expert Rev Med Devices 9:361-366, 2012

3. Ahn Y, Lee HY, Lee SH, Lee JH: Dural tears in percutaneous endoscopic lumbar discectomy. Eur Spine J 20:58-64, 2011

4. Choi G, Lee SH, Bhanot A, Raiturker PP, Chae YS: Percutaneous endoscopic discectomy for extraforaminal lumbar disc herniations: Extraforaminal targeted fragmentectomy technique using working channel endoscope. Spine (Phila Pa 1976) 32:E93-E99, 2007

5. Choi G, Modi HN, Prada N, Ahn TJ, Myung SH, Gang MS, Lee SH: Clinical results of XMR-assisted percutaneous transforaminal endoscopic lumbar discectomy. J Orthop Surg Res 8:14, 2013

6. Cong L, Zhu Y, Tu G: A meta-analysis of endoscopic discectomy versus open discectomy for symptomatic lumbar disk herniation. Eur Spine J 25:134-143, 2016

7. Han IH, Choi BK, Cho WH, Nam KH: The obturator guiding technique in percutaneous endoscopic lumbar discectomy. J Korean Neurosurg Soc 51:182-186, 2012

8. Jasper GP, Francisco GM, Aghion D, Telfeian AE: Technical considerations in transforaminal endoscopic discectomy with foraminoplasty for the treatment of spondylolisthesis: Case report. Clin Neurol Neurosurg 119:84-87, 2014

9. Jasper GP, Francisco GM, Telfeian AE: Transforaminal endoscopic discectomy with foraminoplasty for the treatment of spondylolisthesis. Pain Physician 17: E703-E708, 2014
10. Kitahama Y, Sairyo K, Dezawa A: Percutaneous endoscopic transforaminal approach to decompress the lateral recess in an elderly patient with spinal canal stenosis, herniated nucleus pulposus and pulmonary comorbidities. Asian J Endosc Surg 6:130-133, 2013

11. Komp M, Hahn P, Oezdemir S, Giannakopoulos A, Heikenfeld R, Kasch R, Merk H, Godolias G, Ruetten S: Bilateral spinal decompression of lumbar central stenosis with the fullendoscopic interlaminar versus microsurgical laminotomy technique: A prospective, randomized, controlled study. Pain Physician 18:61-70, 2015

12. Kong L, Shang XF, Zhang WZ, Duan LQ, Yu Y, Ni WJ, Huang Y: Percutaneous endoscopic lumbar discectomy and microsurgical laminotomy: A prospective, randomized controlled trial of patients with lumbar disc herniation and lateral recess stenosis. Orthopade 48:157-164, 2019

13. Lee DY, Lee SH: Learning curve for percutaneous endoscopic lumbar discectomy. Neurol Med Chir (Tokyo) 48:383-388, 388-389, 2008

14. Li Z, Hou S, Shang W, Song K, Zhao H: New instrument for percutaneous posterolateral lumbar foraminoplasty: Case series of 134 with instrument design, surgical technique and outcomes. Int J Clin Exp Med 8:14672-14679, 2015

15. Li ZZ, Hou SX, Shang WL, Cao Z, Zhao HL: Percutaneous lumbar foraminoplasty and percutaneous endoscopic lumbar decompression for lateral recess stenosis through transforaminal approach: Technique notes and 2 years followup. Clin Neurol Neurosurg 143:90-94, 2016

16. Mahajan A, Samuel S, Saran AK, Mahajan MK, Mam MK: Occupational radiation exposure from C arm fluoroscopy during common orthopaedic surgical procedures and its prevention. J Clin Diagn Res 9:C1-C4, 2015

17. Qin H, Huang S, Xu L, Wei P, Jiang J, Xie Z, Luo X, Tan H, Huang W: Radiation exposure and operation time in percutaneous endoscopic lumbar discectomy using fluoroscopy-based navigation system. World Neurosurg 127:e39-e48, 2019

18. Ruetten S, Komp M, Merk H, Godolias G: Full-endoscopic anterior decompression versus conventional anterior decompression and fusion in cervical disc herniations. Int Orthop 33:1677-1682, 2009

19. Ruetten S, Komp M, Merk H, Godolias G: Surgical treatment for lumbar lateral recess stenosis with the full-endoscopic interlaminar approach versus conventional microsurgical technique: A prospective, randomized, controlled study. J Neurosurg Spine 10:476-485, 2009

20. Wu RH, Deng DH, Huang XQ, Shi CL, Liao XQ: Radiation exposure reduction in ultrasound-guided transforaminal percutaneous endoscopic lumbar discectomy for lumbar disc herniation: A randomized controlled trial. World Neurosurg 2019 (Epub ahead of print)

21. Yeung AT, Tsou PM: Posterolateral endoscopic excision for lumbar disc herniation: Surgical technique, outcome, and complications in 307 consecutive cases. Spine (Phila Pa 1976) 27:722-731, 2002 